\title{
Duodenal bulb plasma cells in duodenitis and duodenal ulceration
}

\author{
B B SCOTT, ANNE GOODALL, P STEPHENSON, AND D JENKINS
}

From the Departments of Medicine and Pathology, Lincoln County Hospital, Lincoln

SUMmary Using an immunoperoxidase technique IgA, IgM, IgE and IgG plasma cells were studied in endoscopic duodenal bulb biopsies taken from 14 controls, 25 patients with grade 1 duodenitis (Whitehead classification), 12 patients with grade 2 duodenitis and three with grade 3 duodenitis. The control counts were compared with those in the jejunum and rectum. In addition cell counts were compared in 16 pairs of patients, with and without duodenal ulcer, exactly matched for grade of duodenitis. The control counts were not significantly different from counts in jejunum or rectum except for IgG which were higher in the jejunum $(p=0.03)$. IgA plasma cell counts were significantly increased in both grade 1 and grade 2 duodenitis compared with controls $(\mathrm{p}<0.05$ and $\mathrm{p}<0.01)$. There was no significant difference for the other plasma cells. All plasma cell counts were decreased in the small group of grade 3 duodenitis compared with the other groups. There was no significant difference between counts in duodenitis whether or not there was associated duodenal ulceration. The isolated $\operatorname{IgA}$ plasma cell response of the duodenal bulb mucosa in duodenitis is very different from that of the jejunal mucosa in coeliac disease, and the rectal mucosa in inflammatory bowel disease and bacterial colitis and probably represents the basic response to any mucosal damage.

Little is known of the aetiology and pathogenesis of duodenitis. It may be part of the spectrum of duodenal ulcer disease ${ }^{1}$ and therefore damage by acid might be important. Although the small intestine is an important immunological organ, no important role has been attached to immunological mechanisms in the pathogenesis of either duodenitis or duodenal ulcer. We have previously shown ${ }^{2}$ significant changes in the plasma cells in duodenojejunal mucosa of patients with coeliac disease - a condition in which immunological mechanisms are thought to play an important part. We therefore thought it would be important to see if there were similar changes in duodenitis, which would suggest that the changes were simply a non-specific effect of mucosal damage. It is not known if plasma cell counts vary according to the site in the small intestine and it was therefore necessary to establish a control range for the duodenal bulb. This also enabled us to compare plasma cell counts at three levels in the gastrointestinal tract using our previous

Address for correspondence: Dr B B Scott, Lincoln County Hospital, Sewell Road, Lincoln LN2 5QY.

Received for publication 9 November 1984 published findings at the duodenojejunal junction ${ }^{2}$ and the rectum ${ }^{3}$. Unfortunately there is no satisfactory definition of duodenitis but the histological classification of Whitehead ${ }^{4}$ is widely used and we therefore defined normality and duodenitis according to that classification. We also took the opportunity of comparing plasma cell counts in duodenitis with and without duodenal ulceration to help determine whether there is any difference between the inflammation in the two conditions.

\section{Methods}

SUBJECTS

\section{Controls}

Fourteen patients who underwent routine upper gastrointestinal endoscopy for a variety of reasons including abdominal pain and diarrhoea. They all had normal looking duodenal bulbs and biopsies from the bulb were all grade 0 in the Whitehead classification. Most were eventually diagnosed as irritable bowel. The mean age was 53 years. There were six men. 
Duodenitis grade 1

Twenty five patients who underwent routine endoscopy, mainly for abdominal pain, whose duodenal bulb biopsies showed grade 1 duodenitis in the Whitehead classification - that is, increased lamina propria cellularity but normal general morphology of surface epithelium. Seven had an ulcer in the bulb and in nine the bulb appeared normal at endoscopy. The mean age was 64 years. There were 16 men.

\section{Duodenitis grade 2}

Twelve patients who underwent routine endoscopy, mainly for abdominal pain, whose duodenal bulb biopsies showed grade 2 duodenitis - that is, an abnormality of the surface epithelium. Four had an ulcer in the bulb and in three the bulb appeared normal at endoscopy. The mean age was 48 years. There were eight men.

\section{Duodenitis grade 3}

Three patients who underwent routine endoscopy for abdominal pain whose duodenal bulb biopsies showed grade 3 duodenitis - that is, erosions of the surface epithelium. Two had an ulcer in the bulb. The mean age was 56 years. There were two men.

\section{Duodenal ulcer versus non-ulcer duodenitis}

Sixteen patients with an ulcer (mean age 62 years; 12 men) were exactly matched for duodenitis grade with 16 patients without an ulcer (mean age 57 years; $11 \mathrm{men}$ ). All these patients except three with ulcers are included in the previous group.

\section{BIOPSY}

Endoscopy was done with an Olympus GIF-T endoscope in the majority and an Olympus GIF-Q endoscope in the remainder. Biopsies were taken from the duodenal bulb using either FB-13K or FB-24K forceps. The biopsies were removed from the forceps by shaking in Ringer's solution, orientated on plastic mesh and fixed in formalin as previously described. ${ }^{5}$ Only biopsies which were well orientated and of adequate size were used.

\section{STAINING TECHNIQUES}

The fixed specimens were embedded into paraplast. Tissue sections were cut at $1 \mu$ thickness perpendicular to the luminal surface, mounted on glass slides and dried overnight at $37^{\circ} \mathrm{C}$. Sections were stained for IgA, IgM, IgE, and IgG by the PAP immunoperoxidase technique $^{6}$ after initial trypsinisation ${ }^{7}$ and counterstained with Harris haematoxylin for the morphometric study. Rabbit anti-human immunoglobulin heavy chain antisera, together with swine anti-rabbit antiserum, and peroxidase-rabbit antiperoxidase complex were obtained from Dakopatts.
Specificity of the primary antisera was confirmed by immunoelectrophoresis and by testing against human monoclonal myeloma cases. Particular care was taken to standardise the anti-IgE antiserum using positive and negative substrates. We used a single batch of anti-IgE antiserum for the whole study and no cross reactivity was detected with this batch on control sections. Titration of primary antisera in the range 1:50 to 1:32 000 showed that at high dilutions the count of immunoglobulin containing cells fell with decreasing titre. In the intermediate range consistent cell counts could be shown graphically to lie on a plateau. At high concentration non-specific staining made identification of cellular immunoglobulin very inaccurate. For these reasons cell counts were made within the plateau range. This was found to vary from case to case and the titration was therefore carried out for each case to obtain the plateau count.

\section{MEASUREMENTS}

Measurements and cell counts on the immunoperoxidase stained sections were carried out using a MOPPET image analyser. An image of the stained sections was projected at standard magnification on to the digitising tablet by a Leitz Neopromar projection microscope. The MOP stylus was fitted with a ball point pen to provide a permanent record of the field examined. Four contiguous, nonoverlapping fields were examined on each section. A field was defined as the area between the mucosal surface and muscularis mucosae bounded by two lines perpendicular to the muscularis mucosae. The following three measurements were made on each field: (1) mucosal length - the length of the muscularis mucosae; (2) area of lamina propria; and (3) immunoglobulin containing cell counts. A micrometer slide was used to convert area and length measurements on the projected image into absolute measurement. For each section cell counts were expressed as cells $/ \mathrm{mm}$ mucosal length. All measurements and processing were performed blind on randomised specimens to minimise experimental and observer bias.

\section{STATISTICAL METHODS}

Immunoglobulin containing cell counts in each group were compared using the Student's $t$ test with Bessel's correction for small samples and, for IgG and IgM, log transformation.

\section{Results}

The plasma cell counts are shown in Figs. 1-4. The mean counts for $\operatorname{IgA}$, IgM, IgE, and IgG plasma cells in the controls were $276,100,114$, and 41 


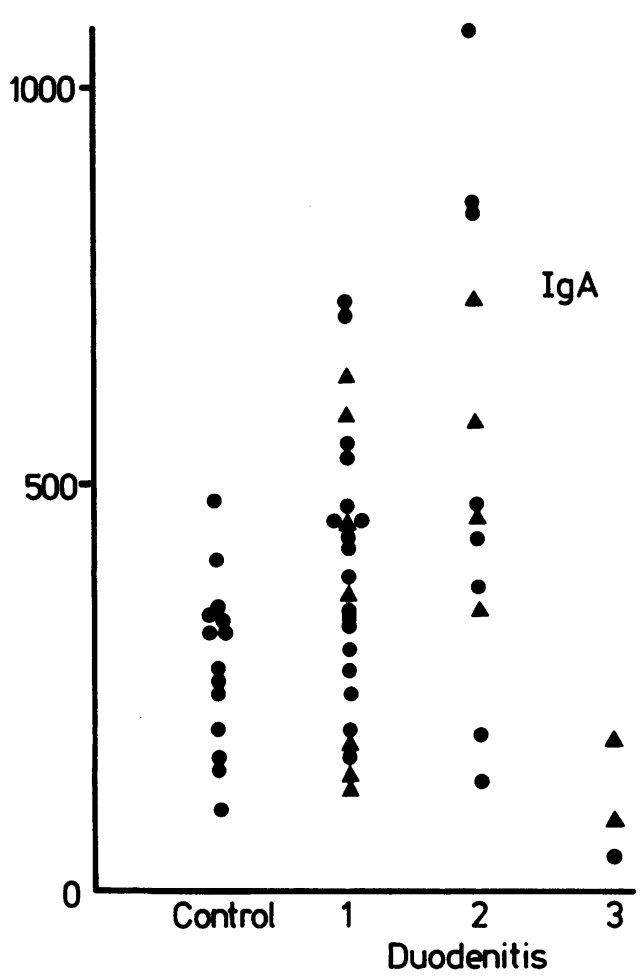

Fig. 1 IgA plasma cell counts/mm mucosal length. The triangles represent patients with a duodenal ulcer.

respectively. The results are very similar to those from the duodenojejunal junction and rectum using our previous published results. ${ }^{2}{ }^{3}$ The only significant difference was for IgG cells which were significantly higher in the duodenojejunal junction $(p=0.03)$. The observed ranges and the means at the three sites are shown in Figs. 5-8. It can be seen that rectal counts are significantly lower than duodenojejunal counts.

In duodenitis grades 1 and $2 \operatorname{IgA}$ plasma cell counts were significantly higher than controls ( $p<0.05$ and $p<0.01$ respectively). IgE, IgM, and IgG plasma cell counts were not significantly different. All the counts in grade 3 duodenitis were lower than controls and although this was significant for $\operatorname{IgA}, \operatorname{IgE}$ and $\operatorname{IgM}(p=0.02,0.01$ and 0.04 respectively) the group was very small. No significant difference could be detected between the groups with or without duodenal ulceration.

\section{Discussion}

The results of this and our previous studies show

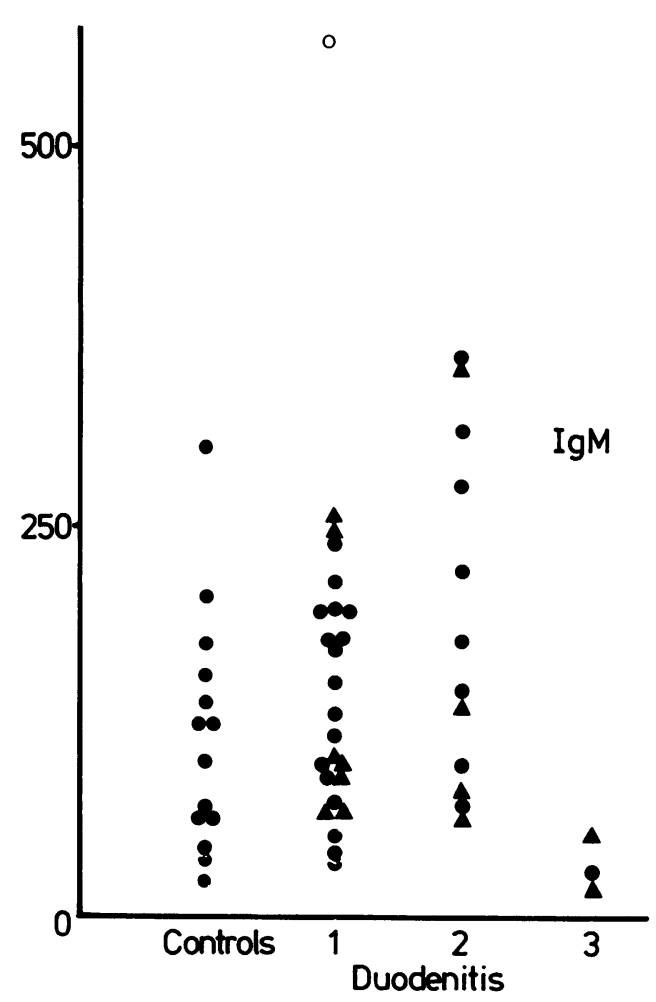

Fig. 2 IgM plasma cell counts/mm mucosal length. The triangles represent patients with a duodenal ulcer. The open circle represents one patient with a count of 920.

that in control populations, judged normal by histology, and where accessible, endoscopy, plasma cell counts are higher in the small intestine than the rectum. The counts tend to be lower in the duodenal bulb than at the duodenojejunal junction. Accurate comparisons at different sites are, however, difficult to make because of possible differences in shrinkage related to fixation of specimens of different shapes and surface area and also because of differences brought about by distortion of the specimens by different biopsy instruments. The mean ages of the three control groups varied from 38-53 years but we have found no significant correlation between plasma cell counts and age within the range studied. Another study ${ }^{8}$ has shown the paucity of IgA cells in normal gastric body mucosa where, given the acid conditions, secretory immunity might not be necessary. The lower level of $\operatorname{IgA}$ cells in the duodenal bulb than in the jejunum might reflect the protective effect of normal levels of gastric acid.

In both mild and moderate duodenitis, in which the structural damage to the mucosa at light microscopic level is minimal, this study shows that 


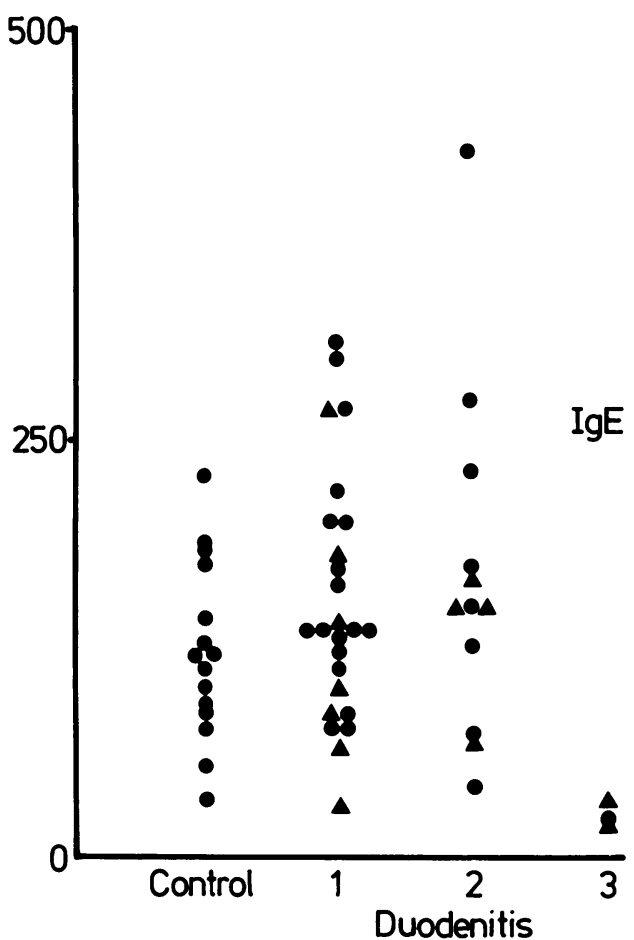

Fig. 3 IgE plasma cell counts/mm mucosal length. The triangles represent patients with a duodenal ulcer.

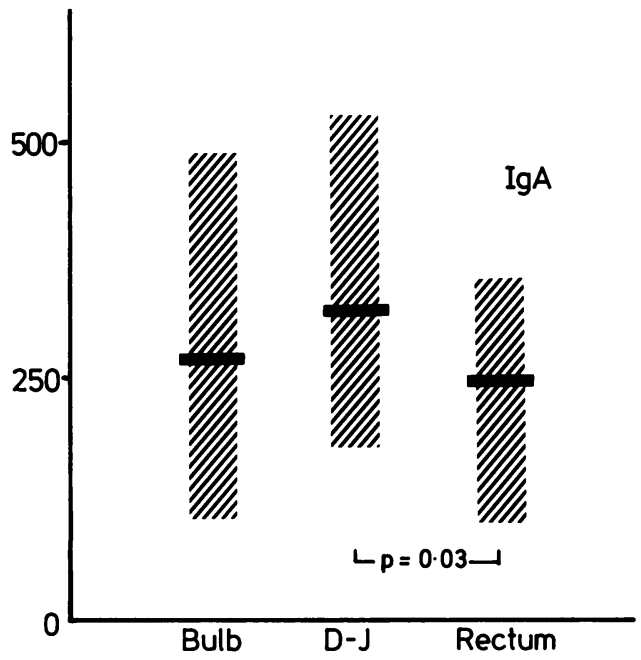

Fig. 5 Observed ranges and means of IgA plasma cell counts $/ \mathrm{mm}$ mucosal length for controls in the duodenal bulb, the duodenojejunal junction (D-J) and the rectum. Significant differences are indicated.

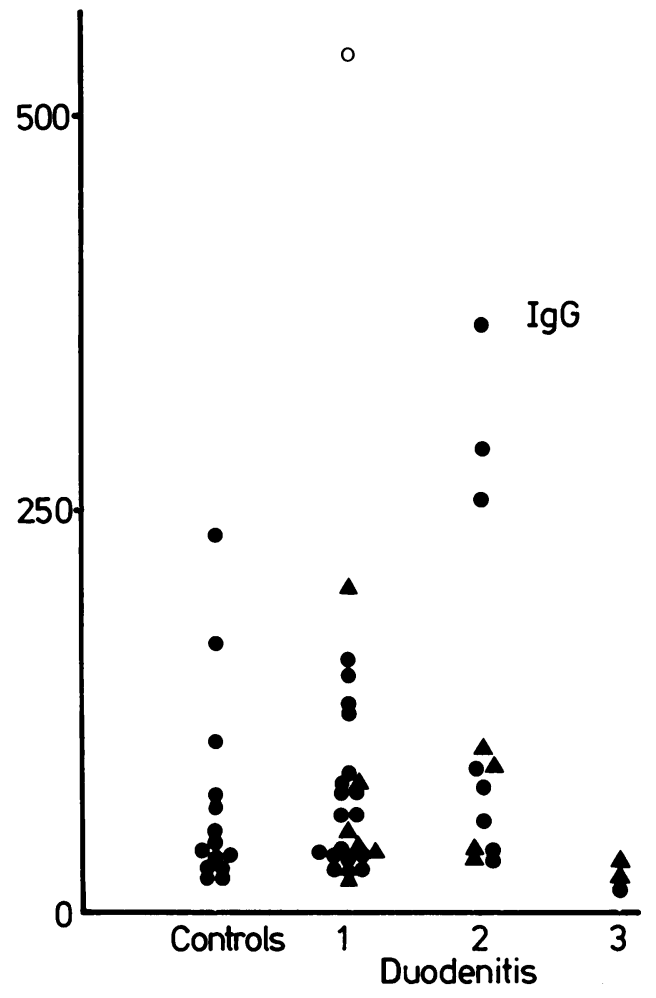

Fig. 4 IgG plasma cell counts/mm mucosal length. The triangles represent patients with a duodenal ulcer. The open circle represents one patient (the same as inFig. 2) with a count of 657.

the humoral response is predominantly IgA. In our three patients with severe duodenitis, in which microscopic ulceration, loss of villi, and the presence of many reactive fibroblasts and endothelial cells indicated structural damage, there was a decrease in the number of all types of plasma cells although the total cellularity and number of inflammatory cells was increased. These findings indicate a different pattern of plasma cell response to that observed in coeliac disease ${ }^{2}$ (in which $\operatorname{Ig} \mathrm{A}, \operatorname{IgM}$, and IgE plasma cells are increased) and in the rectum in inflammatory bowel disease (in which all plasma cells are increased) and in bacterial colitis (in which $\operatorname{IgA}$ and IgE plasma cells are increased). ${ }^{3}$ The aetiology of duodenitis is uncertain but the histological similarity to changes in the duodenum adjacent to peptic ulceration and the response to Cimetidine therapy suggests that acid may have an important role. ${ }^{10}$ Our observation that the plasma cell response is similar in duodenitis with or without ulceration gives further support to the similarity of 


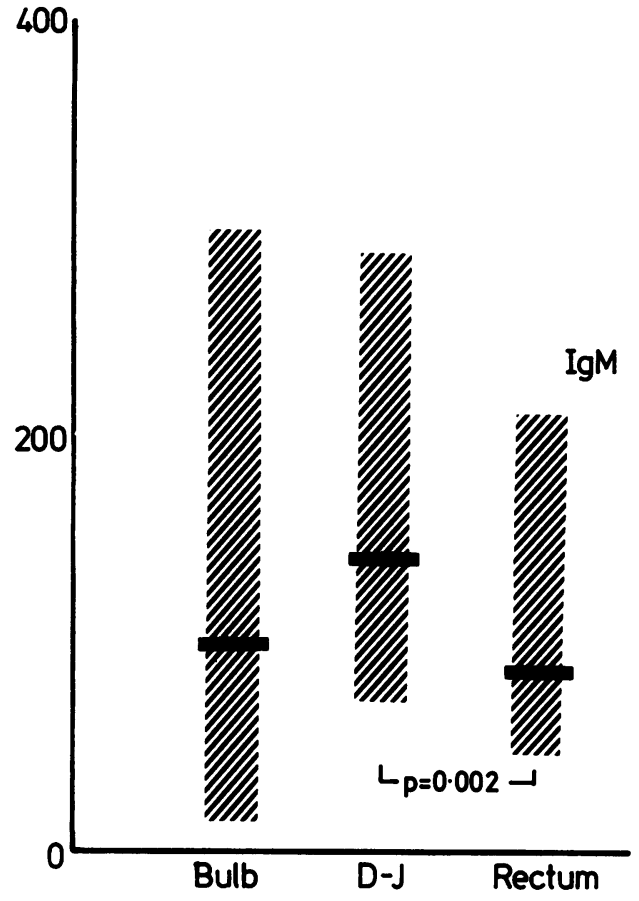

Fig. 6 The observed ranges and means of IgM plasma cell counts $/ \mathrm{mm}$ mucosal length for controls in the duodenal bulb, the duodenojejunal junction (D-J) and the rectum. Significant differences are indicated.

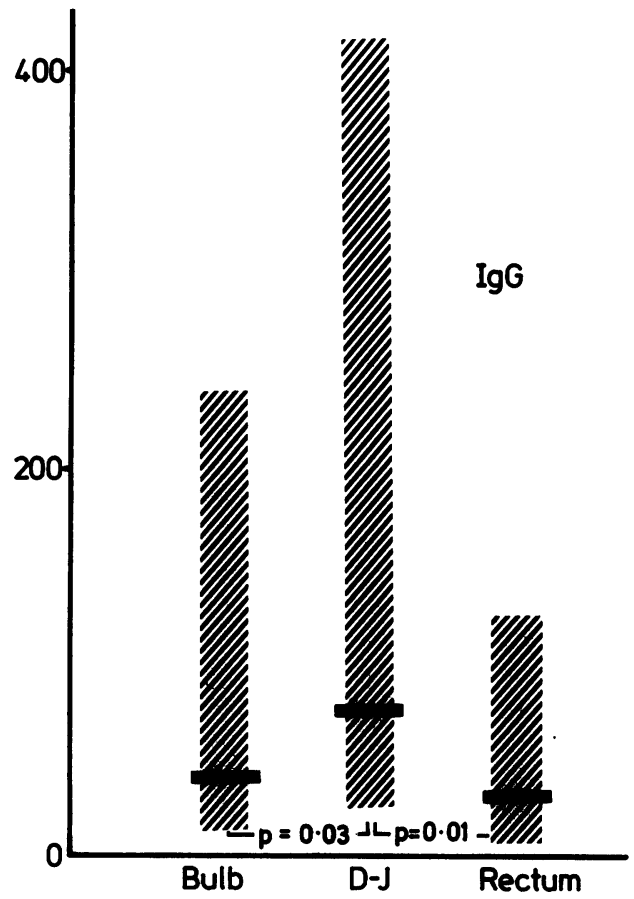

Fig. 8 Observed ranges and means of IgG plasma cell counts/mm mucosal length for controls in the duodenal bulb, the duodenojejunal junction (D-J) and the rectum. Significant differences are indicated.

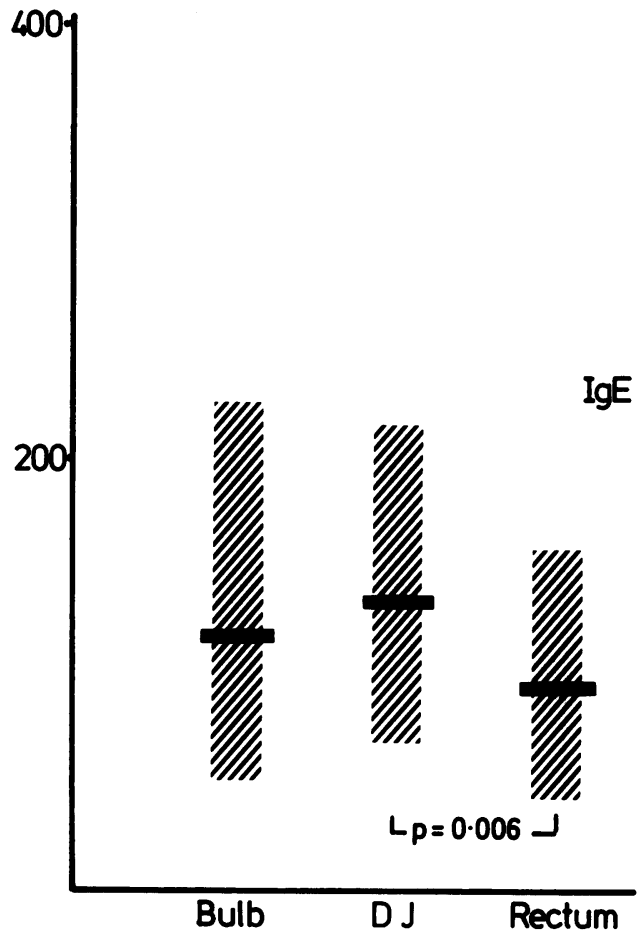

Fig. 7 Observed ranges and means of IgE plasma cell counts $/ \mathrm{mm}$ mucosal length for controls in the duodenal bulb, the duodenojejunal junction (D-J) and the rectum. Significant differences are indicated.

the two conditions.

Whereas an IgA response seems to occur in all types of intestinal disease studied, duodenitis is the only condition in which an isolated increase in IgA plasma cells has been shown. This response may be explained by what is known of the biological role of IgA. It has recently been suggested that the primary role of $\operatorname{IgA}$ is to clear soluble and small particulate material that crosses the epithelium of mucosal surfaces. ${ }^{11}$ The intestinal epithelium, even in the normal state is not a complete barrier to antigenic macromolecules. Although the effect of non-specific duodenitis on permeability of the duodenum to macromolecules is not known, it is known that ethanol, which can produce a clinically and pathologically similar duodenitis, increases intestinal absorption of the macromolecule horseradish peroxidase, apparently by destabilising the intercellular junctions of the epithelium and allowing penetration of horseradish peroxidase between the enterocytes. ${ }^{12}$ It is possible that there is a similar increased permeability to antigenic macromolecules in nonspecific duodenitis. At sites of microscopic ulceration in severe duodenitis, structural damage to the mucosa may interfere with recirculation of plasma cell precursors.

The response in duodenitis is clearly different 
from that seen in coeliac disease and inflammatory bowel disease where there may be disorders of immune regulation, and from that seen in response to known infectious agents. The fact that there is more than just an $\operatorname{IgA}$ response in coeliac disease, inflammatory bowel disease and bacterial colitis suggest that the plasma cell response in each of those conditions is not simply a non-specific effect of mucosal damage but that it is also either intimately involved in pathogenesis or a specific response to certain antigens or microorganisms.

We thank the Trent Regional Health Authority Research Committee for generous financial support.

\section{References}

1 Spiro HM. Moynihan's disease? The diagnosis of duodenal ulcer. $N$ Engl J Med 1974; 291: 567-9.

2 Scott BB, Goodall A, Stephenson P, Jenkins D. Small intestinal plasma cells in coeliac disease. Gut 1984; 25: 41-6.

3 Scott BB, Goodall A, Stephenson P, Jenkins D. Rectal mucosal plasma cells in inflammatory bowel disease. Gut 1983; 24: 519-24.
4 Whitehead R, Roca M, Meikle DD, Skinner J, Truelove SC. The histological classification of duodenitis in fibreoptic biopsy specimens. Digestion 1975; 13: 129-36.

5 Scott BB, Losowsky MS. Peroral small-intestinal biopsy: experience with the hydraulic multiple biopsy instrument in routine clinical practice. Gut 1976; 17: 740-3.

6 Taylor CR. Immunoperoxidase techniques. Arch Pathol Lab Med 1978; 102: 113-21.

7 Curran RC, Gregory J. The unmasking of antigens in paraffin sections by trypsin. Experientia (Basle) 1977; 33: $1400-1$.

8 Isaacson P. Immunoperoxidase study of the secretory immunoglobulin system and lysozyme in normal and diseased gastric mucosa. Gut 1982; 23: 578-88.

9 Hasan M, Sircus W, Ferguson A. Duodenal mucosal architecture in non-specific and ulcer-associated duodenitis. Gut 1981; 22: 637-41.

10 Hasan M, Hay F, Sircus W, Ferguson A. Nature of the inflammatory cell infiltrate in duodenitis. J Clin Pathol 1983; 36: 280-8.

11 Bienenstock J, Befus AD. Some thoughts on the biological role of immunoglobulin A. Gastroenterology 1983; 84: 178-85.

12 Draper LR, Gyure LA, Hall JG, Robertson D. Effect of alcohol on the integrity of the intestinal epithelium. Gut 1983; 24: 399-404. 